\title{
LINEAR EXTREMAL PROBLEMS FOR HARMONIC MAPPINGS OF THE DISK
}

\author{
PETER DUREN AND GLENN SCHOBER
}

(Communicated by Irwin Kra)

\begin{abstract}
Sharp bounds for Fourier coefficients and distortion are established for harmonic mappings of the unit disk onto itself.
\end{abstract}

\section{INTRODUCTION}

In a previous paper [1], we developed a variational method for treating extremal problems over families of sense-preserving univalent harmonic functions which map the unit disk onto a given convex region. We applied the method to obtain general necessary conditions for the solutions to linear extremal problems. Specializing to harmonic self-mappings of the disk, we then used the results to give the sharp estimates

$$
\left|c_{-n}\right| \leq \frac{n+1}{n \pi} \sin \frac{\pi}{n+1}, \quad n=1,2, \ldots,
$$

for the Fourier coefficients of negative index in the expansion

$$
f\left(r e^{i \varphi}\right)=\sum_{n=-\infty}^{\infty} c_{n} r^{|n|} e^{i n \varphi} .
$$

The "extremal functions" were found to map the disk onto the interior of a regular $(n+1)$-gon inscribed in the unit circle.

We now continue this investigation of harmonic self-mappings of the disk by giving the sharp bounds for the coefficients $c_{n}$ of positive index. Here the problem is essentially different and the extremal functions will be seen to map the disk onto itself. We also treat the problem of maximum distortion by finding the sharp bounds for $\partial f / \partial z, \partial f / \partial \bar{z}, \partial f / \partial x$, and $\partial f / \partial y$ at a prescribed point in the disk. At the origin this is equivalent to estimating $c_{1}, c_{-1}, c_{1}+c_{-1}$, and $c_{1}-c_{-1}$. We then generalize the problem in a different way by estimating $c_{n}+e^{i \alpha} c_{-n}$.

Received by the editors July $18,1988$.

1980 Mathematics Subject Classification (1985 Revision). Primary 30C70.

This work was supported in part by the National Science Foundation under Grants DMS-8601488 and DMS-8601498. 
Before considering these extremal problems, it is necessary to recall a general result obtained in [1]. Let $\mathscr{F}$ be the closure of the family of all orientationpreserving harmonic mappings of the unit disk $\mathbf{D}$ onto itself. Then each $f \in \mathscr{F}$ has a unique Poisson integral representation

$$
f(z)=\frac{1}{2 \pi} \int_{0}^{2 \pi} \frac{1-|z|^{2}}{\left|e^{i t}-z\right|^{2}} e^{i \vartheta(t)} d t,
$$

where $\vartheta$ is a circle mapping, defined as a left-continuous nondecreasing function on $[0,2 \pi)$ with $\vartheta(2 \pi-0)-\vartheta(0) \leq 2 \pi$. In fact, $\mathscr{F}$ is precisely the family of all functions $f$ of the form (2). Each continuous linear functional $L$ on $\mathscr{F}$ has the form

$$
L(f)=\int_{0}^{2 \pi} l(t) e^{i \theta(t)} d t, \quad f \in \mathscr{F},
$$

where $f$ is given by (1) and

$$
l(t)=\frac{1}{2 \pi} L\left(\frac{1-|z|^{2}}{\left|e^{i t}-z\right|^{2}}\right) .
$$

The following theorem was proved in [1] by a variational method.

Theorem A. Let $L$ be a continuous linear functional, let $l$ be defined by (3), and let $f$ maximize $\operatorname{Re}\{L\}$ over $\mathscr{F}$. Then the circle mapping $\vartheta$ which represents $f$ by the formula (2) has the properties:

(a) $\vartheta$ is constant on any interval where $\operatorname{Im}\left\{l e^{i \vartheta}\right\}$ has constant sign;

(b) $\operatorname{Im}\left\{l e^{i \theta}\right\}$ has mean value zero on any interval whose endpoints are discontinuities of $\vartheta$.

\section{CoEfFicients With POSITIVE INDEX}

We now turn to the linear problem of maximizing $\operatorname{Re}\left\{c_{n}\right\}$ for $n=1,2, \ldots$, where $c_{n}$ is the $n$th coefficient in the expansion (1) of a harmonic self-mapping of the disk. This is clearly equivalent to the problem of maximizing $\left|c_{n}\right|$. The associated function $l$ defined in (3) has the form

$$
l(t)=\frac{1}{2 \pi} e^{-i n t},
$$

and we are to maximize

$$
\operatorname{Re}\left\{c_{n}\right\}=\frac{1}{2 \pi} \int_{0}^{2 \pi} \cos [\vartheta(t)-n t] d t
$$

among all circle mappings $\vartheta$. In order to apply the necessary conditions of Theorem A, we note that

$$
\operatorname{Im}\left\{l(t) e^{i \vartheta(t)}\right\}=\frac{1}{2 \pi} \sin [\vartheta(t)-n t]
$$

vanishes on the oblique lines $\theta=n t+k \pi, k=0, \pm 1, \pm 2, \ldots$, shown in Figure 1. On these critical lines $\cos (\theta-n t)$ is alternately equal to \pm 1 , as 


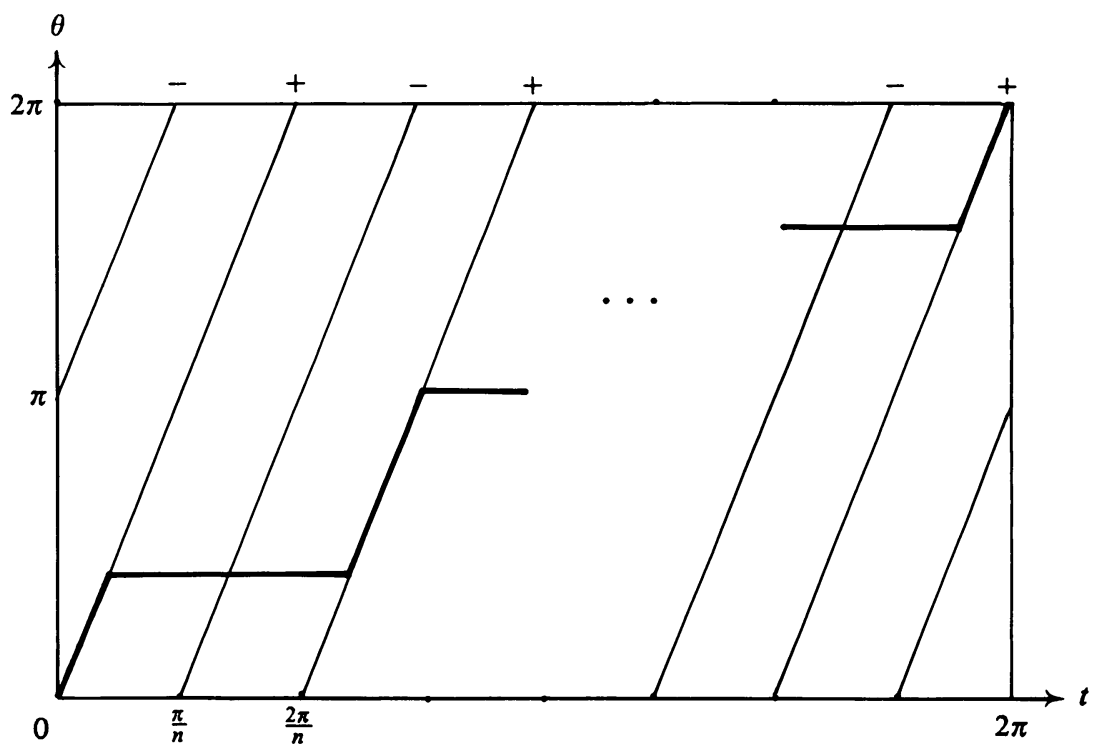

FigURE 1

indicated in the figure. For convenience, a line where $\cos (\theta-n t) \equiv+1$ is called a positive line, while a line on which $\cos (\theta-n t) \equiv-1$ is called a negative line.

Now let $\vartheta$ be a circle mapping which maximizes the integral (4). According to Theorem A, this extremal function $\vartheta$ must be constant in any strip between successive critical lines. Thus $\vartheta$ can increase only along a critical line, or by jumping from one strip to another. It is clear that $\vartheta$ cannot follow a negative line in any interval, because a small perturbation would then increase the integral (4). We conclude that $\vartheta$ can increase only by following a positive line or by jumping. The two endpoints of a jump can never lie in the same (closed) strip.

We may view the graph of $\vartheta$ as a continuous curve if we join the endpoints of each jump by a vertical line. If this curve meets the same positive line at two distinct points, then it must follow the line in the corresponding interval. Otherwise, $\vartheta$ would not maximize the integral (4).

In view of the periodicity of the integrand, we may assume without loss of generality that $\vartheta(0)=0$. Indeed, if $0<\vartheta(0)<2 \pi$, then there is some point $t_{0}$ where $\vartheta\left(t_{0}\right)=n t_{0}$, and the circle mapping $\tilde{\vartheta}$ defined by

$$
\tilde{\vartheta}(t)=\vartheta\left(t+t_{0}\right)-n t_{0}, \quad \text { where } \vartheta(t+2 \pi)=\vartheta(t)+2 \pi,
$$

gives rise to the same value of the integral (4). Thus we may suppose that the graph of $\vartheta$ climbs from $(0,0)$ to $(2 \pi, 2 \pi)$, as shown in Figure 1.

Next, we claim that $\vartheta$ is a continuous function. If its graph jumps over a positive line, then it must meet the same line later, which as we have seen is impossible. For the same reason a jump cannot start from a positive line. 

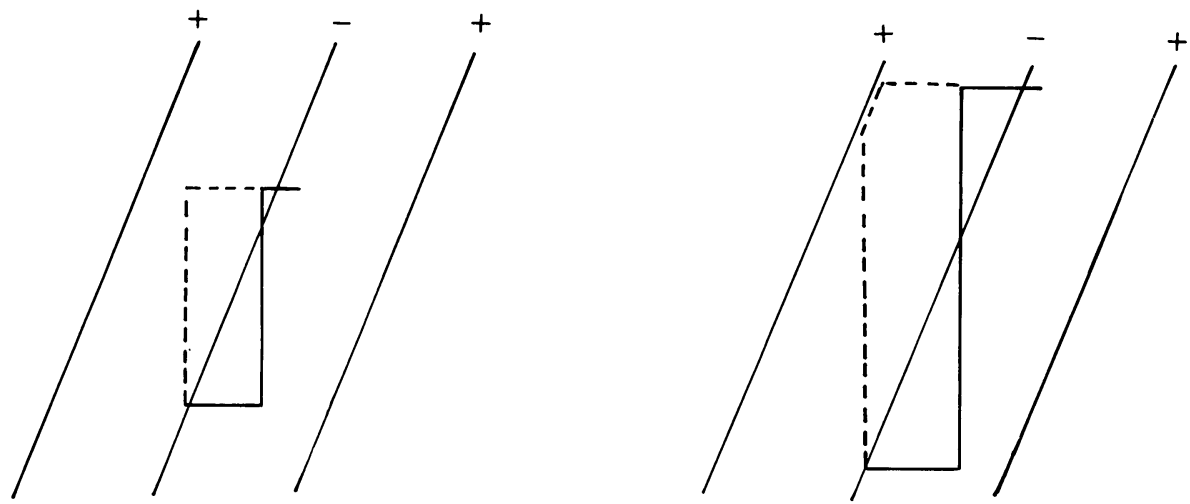

FIGURE 2

Assume now that the graph jumps over a negative line. Then it must have met the same negative line earlier. There are two cases, as shown in Figure 2.

In either case, the graph can be modified to increase the value of (4), as shown by the dashed lines in Figure 2.

Thus the extremal function $\vartheta$ is continuous, and it increases only along positive lines. Its intervals of constancy have lengths that are integer multiples of $2 \pi / n$. These intervals contribute zero to the integral (4). The intervals of increase have total length $2 \pi / n$, and so $\operatorname{Re}\left\{c_{n}\right\}=1 / n$ in the extremal case. We have proved the following theorem.

Theorem 1. Let $f$ be a sense-preserving univalent harmonic mapping of the unit disk onto itself, with the Fourier expansion (1). Then $\left|c_{n}\right| \leq 1 / n, n=1,2 \ldots$. For $n=1$, equality occurs if and only if $f$ is a rotation: $f(z)=e^{i \alpha} z$. For $n \geq$ 2 , equality occurs if and only if $f$ is generated by a continuous, piecewise linear circle mapping $\vartheta$ which has intervals $(\bmod 2 \pi)$ of constancy whose lengths are integer multiplies of $2 \pi / n$, alternated with intervals of increase where $d \vartheta / d t=$ $n$. In all cases the extremal functions map the unit disk onto itself.

\section{Distortion}

As a further application of the basic criterion for solutions to linear extremal problems (Theorem A), we shall now consider the problem of maximal distortion at a given point under an arbitrary harmonic mapping of the disk onto itself. In the absence of the Cauchy-Riemann equations, it is not clear how the distortion should be measured. Two natural candidates are the Jacobian and the norm of the gradient, but these present nonlinear problems to which our methods are not directly applicable. Instead, we shall consider the problem of bounding the derivatives $\partial f / \partial z, \partial f / \partial \bar{z}, \partial f / \partial x$ and, $\partial f / \partial y$.

Given a point $\zeta \in \mathbf{D}$, we first pose the problem of maximizing $\operatorname{Re}\left\{f_{z}(\zeta)\right\}$ over the class $F$ of orientation-preserving harmonic mappings $f$ of $\mathbf{D}$ onto 
itself. This is a linear problem with kernel

$$
l(t)=\frac{1}{2 \pi} e^{i t}\left(e^{i t}-\zeta\right)^{-2} .
$$

Thus the critical curves $\operatorname{Im}\left\{l e^{i \vartheta}\right\}=0$ are described by the equation

$$
\operatorname{Im}\left\{e^{i(\theta-t)}\left(1-\zeta e^{-i t}\right)^{-2}\right\}=0 .
$$

Because the Koebe function $k(z)=z(1-z)^{-2}$ is starlike with respect to the origin, the equation (5) is easily seen to determine $\theta=\vartheta(t)$ as an increasing function of $t$. Here we are implicitly excluding the case $\zeta=0$, which gives again the problem of maximizing $\operatorname{Re}\left\{c_{1}\right\}$. We now make the direct estimate

$$
\begin{aligned}
\operatorname{Re}\left\{f_{z}(\zeta)\right\}=\frac{1}{2 \pi} \int_{0}^{2 \pi} \operatorname{Re}\left\{\frac{e^{i[\theta(t)-t]}}{\left(1-\zeta e^{-i t}\right)^{2}}\right\} & d t \\
& \leq \frac{1}{2 \pi} \int_{0}^{2 \pi} \frac{d t}{\left|1-\zeta e^{-i t}\right|^{2}}=\frac{1}{1-|\zeta|^{2}} .
\end{aligned}
$$

Equality occurs if and only if (5) is satisfied and the real part of the same expression is positive. As we have already observed, this uniquely determines a circle mapping $\theta=\vartheta(t)$ generating the extremal function, which maps the disk univalently onto itself. In particular, the estimate is sharp.

Alternatively, we may argue as follows. If a function $f \in \mathscr{F}$ is precomposed with a Moebius self-mapping of the disk, we obtain a function $g \in \mathscr{F}$ defined by

Observe that

$$
g(z)=f\left(\frac{z+\zeta}{1+\bar{\zeta} z}\right), \quad \zeta \in \mathbf{D} .
$$

$$
g_{z}(0)=f_{z}(\zeta)\left(1-|\zeta|^{2}\right)
$$

and

$$
g_{z}(0)=f_{\bar{z}}(\zeta)\left(1-|\zeta|^{2}\right)
$$

Thus the sharp inequalities $\left|c_{1}\right| \leq 1$ and $\left|c_{-1}\right|<2 / \pi$ lead at once to sharp bounds for $f_{z}(\zeta)$ and $f_{z}(\zeta)$. We may summarize our results as follows.

Theorem 2. Let $f$ be a sense-preserving univalent harmonic mapping of the unit disk onto itself, and let $\zeta \in \mathbf{D}$ be a given point. Then the sharp inequalities

$$
\left|f_{z}(\zeta)\right| \leq\left(1-|\zeta|^{2}\right)^{-1}
$$

and

$$
\left|f_{z}(\zeta)\right|<\frac{2}{\pi}\left(1-|\zeta|^{2}\right)^{-1}
$$

hold. The first bound is attained, but the second is not. The "extremal functions" for the second estimate are harmonic mappings of the disk onto a line segment which forms a diameter of the unit disk.

The sharp bounds for $\partial f / \partial x$ and $\partial f / \partial y$ can be obtained by similar methods. It is convenient first to find the bounds for $c_{1} \pm c_{-1}$, then to deduce the 
general estimates as above by a Moebius transformation. Actually, it is almost as easy to estimate $c_{n}+e^{i \alpha} c_{-n}$. We have the following result.

Theorem 3. Let $f$ be a sense-preserving univalent harmonic mapping of the unit disk onto itself, with the Fourier expansion (1). Then the coefficients $c_{n}$ satisfy the sharp inequalities

$$
\left|c_{n}+e^{i \alpha} c_{-n}\right|<\frac{4}{n \pi}, \quad n=1,2, \ldots
$$

Theorem 4. For each point $\zeta \in \mathbf{D}$ the sharp inequalities

$$
\left|f_{x}(\zeta)\right|<\frac{2}{\pi}\left(1-|\zeta|^{2}\right)^{-1}
$$

and

$$
\left|f_{y}(\zeta)\right|<\frac{2}{\pi}\left(1-|\zeta|^{2}\right)^{-1}
$$

hold for all univalent harmonic mappings $f$ of $\mathbf{D}$ onto itself.

Proof of Theorem 4. Choose $n=1$ and $e^{i \alpha}= \pm 1$ in Theorem 3, and use the formulas (6) and (7).

Proof of Theorem 3. Since the rotations $e^{i \beta} f\left(e^{i \gamma} z\right)$ preserve the family, it is sufficient to consider the functional $\operatorname{Re}\left\{c_{n}+c_{-n}\right\}$. Thus let $f \in \mathscr{F}$ maximize

$$
\operatorname{Re}\left\{c_{n}+c_{-n}\right\}=\frac{1}{\pi} \int_{0}^{2 \pi} \cos n t \cos \vartheta(t) d t
$$

for a fixed integer $n \geq 1$. Then the critical curves are given by the equation

$$
\operatorname{Im}\left\{l(t) e^{i \vartheta(t)}\right\}=\operatorname{Im}\left\{\frac{1}{2 \pi}\left(e^{-i n t}+e^{i n t}\right) e^{i \vartheta(t)}\right\}=\frac{1}{\pi} \cos n t \sin \vartheta(t)=0 .
$$

That is, the critical curves consist of the horizontal lines $\theta=k \pi$ and the vertical lines $t=(1 / n)(\pi / 2+k \pi), k=0, \pm 1, \pm 2, \ldots$, as shown in Figure 3 .

It now follows from Theorem A that the extremal circle mapping $\theta=\vartheta(t)$ is piecewise constant, and that in any interval $(a, b)$ between successive jumps either $\vartheta(t) \equiv k \pi, k=0, \pm 1, \pm 2, \ldots$, or $\int_{a}^{b} \cos n t d t=0$. In the latter case the interval $(a, b)$ contributes zero to the integral $(10)$. Thus the only intervals which make nonzero contributions are those for which $\vartheta(t) \equiv k \pi$. Consequently, we may shift the graph of $\vartheta$ on the other intervals of constancy, without affecting the value of the integral, so that $\vartheta$ assumes only the values $0, \pi$, and $2 \pi$. In other words, we may suppose that

$$
\vartheta(t)= \begin{cases}0, & 0<t \leq a, \\ \pi, & a<t \leq b, \\ 2 \pi, & b<t \leq 2 \pi\end{cases}
$$

Then formula (8) gives

$$
\operatorname{Re}\left\{c_{n}+c_{-n}\right\}=\frac{2}{n \pi}[\sin (n a)-\sin (n b)] .
$$




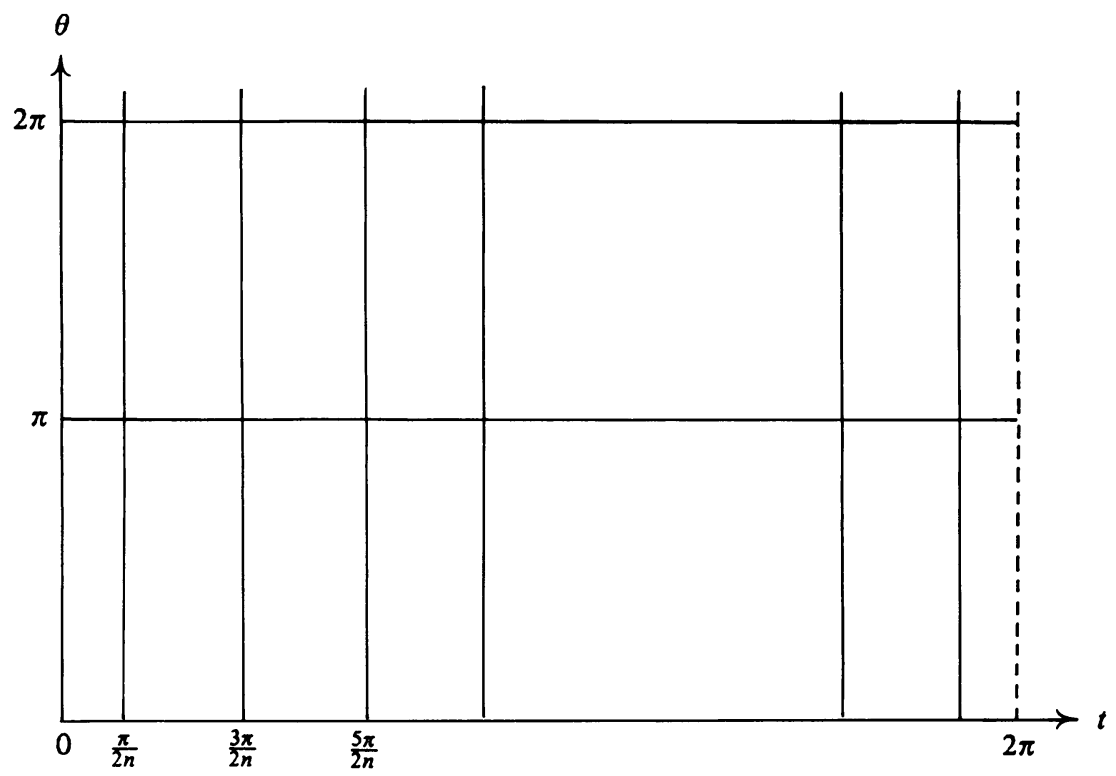

FIGURE 3

Clearly, we can do no better than choose $a=\pi / 2 n$ and $b=3 \pi / 2 n$. This gives the extremal value $\operatorname{Re}\left\{c_{n}+c_{-n}\right\}=4 / n \pi$.

For $n=1$ the only extremal functions are those which map the disk onto a diametral segment of the unit disk. For $n>1$ there are other extremal functions which map the disk onto polygons with at most a certain number of sides, inscribed in the unit circle. In no case is equality attained for a harmonic mapping of the disk onto itself.

\section{REFERENCES}

1. P. Duren and G. Schober, A variational method for harmonic mappings onto convex regions, Complex Variables Theory Appl. 9 (1987), 153-168.

Department of Mathematics, University of Michigan, Ann Arbor, Michigan 48109

Department of Mathematics, Indiana University, Bloomington, Indiana 47405 